\title{
Femtosecond-laser experiment for Master II students: generation, measurement and control of femtoseconds pulses
}

Fréderic Druon, Tom Peyrot, Arthur Larrouy, Arnaud Courvoisier, Cédric Lejeune, et al.

Fréderic Druon, Tom Peyrot, Arthur Larrouy, Arnaud Courvoisier, Cédric Lejeune, Thierry Avignon, "Femtosecond-laser experiment for Master II students: generation, measurement and control of femtoseconds pulses," Proc. SPIE 9793, Education and Training in Optics and Photonics: ETOP 2015, 97930 T (8 October 2015); doi: 10.1117/12.2223086

Event: Education and Training in Optics and Photonics: ETOP 2015, 2015, Bordeaux, France 


\title{
Femtosecond-laser experiment for Master II students: Generation, measurement and control of femtoseconds pulses
}

\author{
Fréderic Druon ${ }^{1,2}$, Tom Peyrot $^{1}$, Arthur Larrouy ${ }^{1}$, Arnaud Courvoisier ${ }^{1}$, Cédric Lejeune ${ }^{1}$, \\ Thierry Avignon ${ }^{1}$ \\ ${ }^{1}$ Institut d'Optique Graduate School \\ 2 Avenue A. Fresnel, 91127 Palaiseau Cedex, France \\ ${ }^{2}$ Laboratoire Charles Fabry, ibid
}

In the framework of the experiment platform LEnsE (Laboratoire d'Enseignement Expérimental) of the Institut d'Optique Graduate School in Palaiseau, we present a new lab work dedicated to Master-II-level students. This lab work is integrated in the formation in the field of ultrashort-pulse lasers and its objective is to train students to this specific technology. The varied topics include generation, measurement and basic control of ultrashort pulses. Key concepts are studied, such as the time-frequency duality, nonlinear effects, the group velocity dispersion (GVD) and more generally managing spectral and temporal phase.

The lab work is based on a totally accessible Ti:sapphire laser (Mira 800 from Coherent). It is used to understand crucial concepts in the generation process such as GVD and self-phase-modulation in the solitonic regime and Kerr lens mode-locking. Because the pulse measurement is a crucial issue to address in ultrafast optics, the lab work also studies different apparatus commonly used to fully characterize fs pulse train: photodiode, spectrometer, and more specifically second-order autocorrelator. The autocorrelation concept is detailed using a homemade accessible apparatus. For a simple manipulation of femtosecond pulses, we propose to realize a spectral-phase control with high-dispersive glass to temporally stretch the pulses. GTI mirrors then re-compress them. The three pillars generation-measurement-control will be described with a practical approach at the conference.

Keywords: femtosecond-laser, ultrashort-pulse, lab work

\section{Introduction : IOGS and LEnsE}

The Institut d'Optique Graduate School (IOGS) is an engineering school in Optics and Photonics whose strength appears in the high correlation between courses and experimental handlings. In each of the three sites of the school that are located in, Palaiseau, Bordeaux and Saint-Etienne, the part of experimental training is crucial. The education received then allows the student to move towards engineering, entrepreneurship and research. Accordingly, the necessity of designing new current issues lab works is part of the school approach success. The LEnsE coordinates the laboratory and the courses. Thanks to a set of 60 teachers, about 1200 hours of experimental training are provided each year. This represents for each student a panel of 68 lab works. Since various topics are developed such as noises in detectors, polarization of the light and laser optics, a graduate student from the institute is introduced to the main techniques of both engineer and researcher. This is in this strong interaction between the LEnsE, the laboratory and the courses that the new femtosecond-laser experiment for Master II is developed.

\section{Femtosecond laser Labwork}

The lab work, we propose is mainly composed of three important axes to understand the technology of femtosecond lasers: the generation, measurement and manipulation of femtosecond pulses. Students will gain knowledge on key concepts in the study of femtosecond lasers, such as the time-frequency relationship,

Education and Training in Optics and Photonics: ETOP 2015, edited by Eric Cormier, Laurent Sarger Proc. of SPIE Vol. 9793, 97930T · (C) 2015 SPIE, IEEE, OSA, ICO · doi: 10.1117/12.2223086 
nonlinear effects, the group velocity dispersion and managing spectral and temporal phases. By this mean we want to fulfill the gap between the theoretical and practical knowledge on these particular subjects.

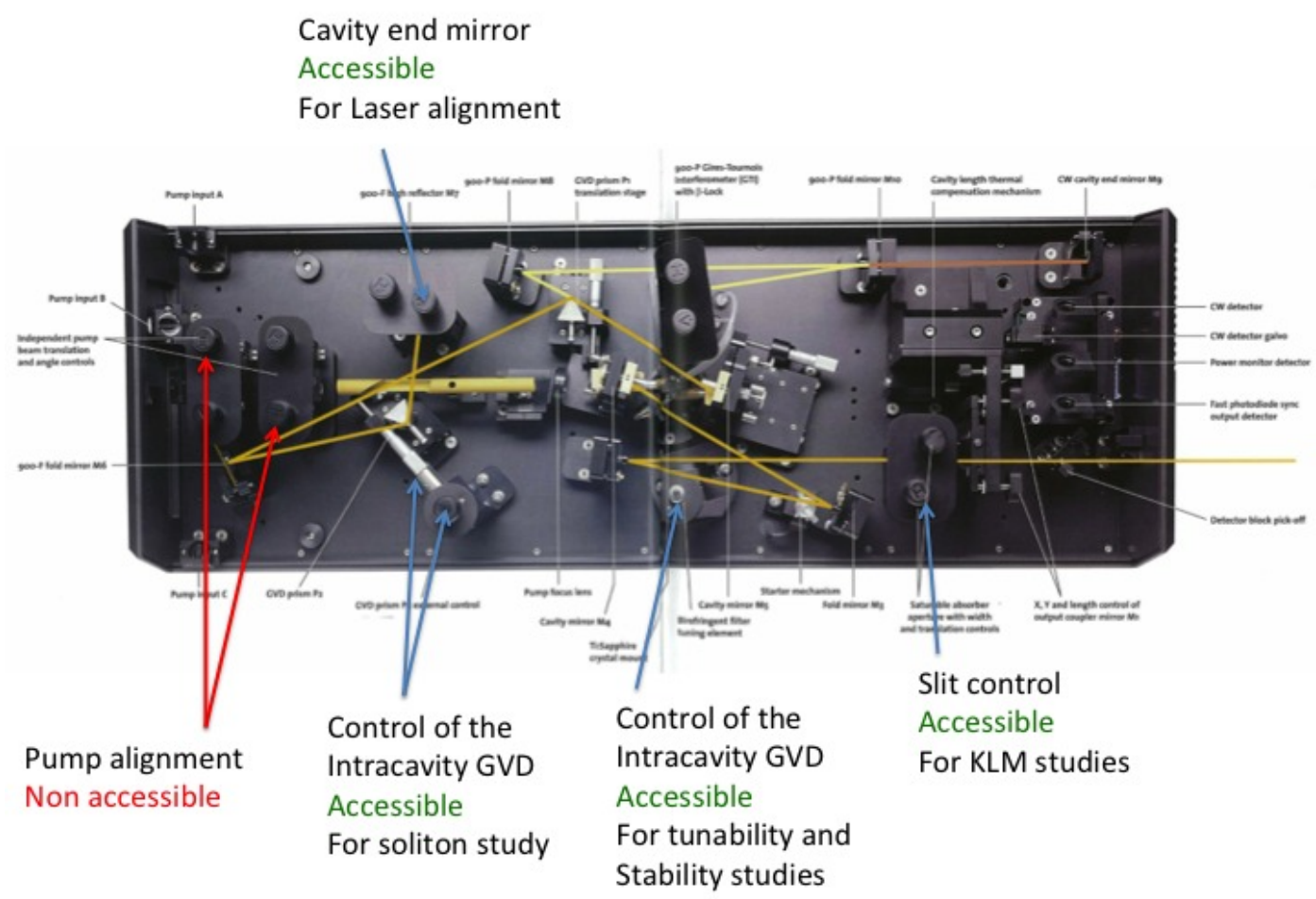

figure 1: picture of the laser with the different knobs accessible for the student. (Courtesy of Jean-Luc Tapié, Coherent)

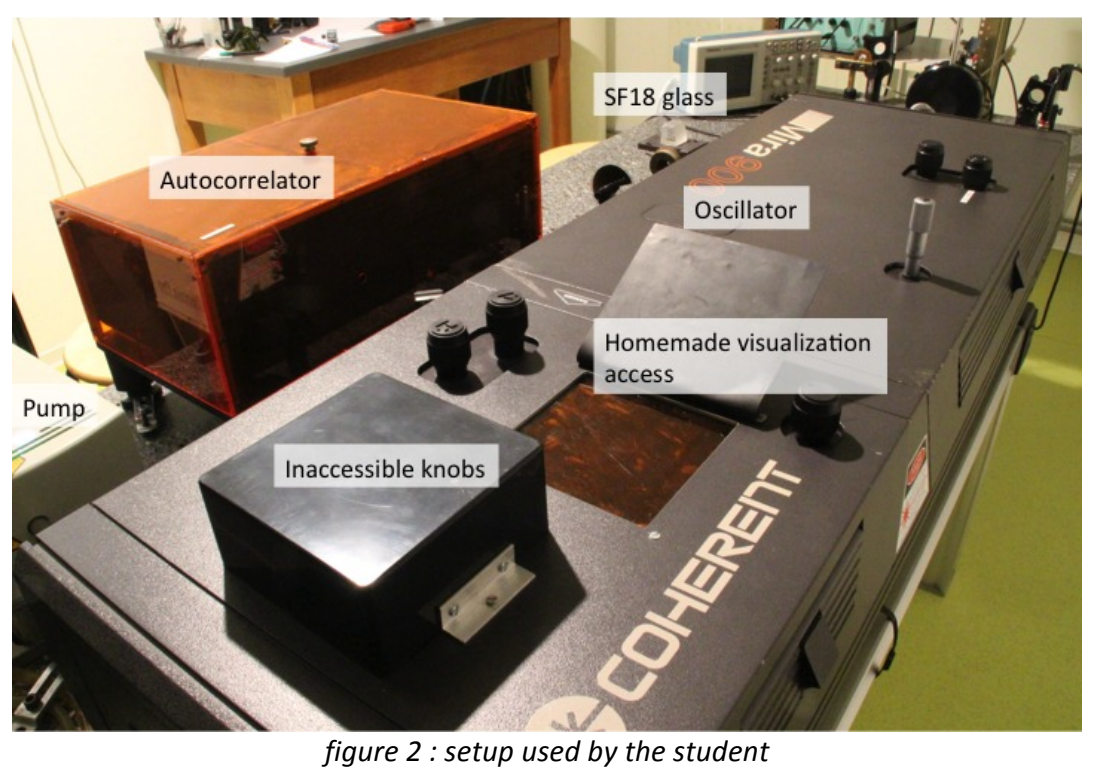

\section{a) On the equipment}

Because we expect the students to get technically familiar with the ultra-short pulses, all the devices used are definitely accessible. Indeed, the two main components -that are the laser and the autocorrelator- have been modified for the student to appropriate the handling. 
For the generation of femtosecond pulses, the lab work uses an oscillator based on technology Ti: Sapphire which is undoubtedly the most classic and current technology in this area-. The laser is a Mira Optima 900-F from Coherent. As shown in figures 1 and 2 representing the oscillator, different knobs are accessible together with their lecture to perform some measurements. All these experiments can be done with the laser covered. This has several advantages, for safety issues: allowing the wearing of goggles only cutting the IR; for reliability issues preserving the laser from the air turbulence and from mistakes on the knob to turn. The teacher has also the possibility to uncover the oscillator to present the setup to the students.

In the same point of view, the SHG autocorrelator can also be open and has full accessibility of this adjustment. Since this autocorrelator is much less sensitive than the oscillator, the autocorrelator can be used covered or uncovered by the students (figure 3). The autocorrelator that is based on a Michelson interferometer has one of its arms on a manual translation stage and the other on the vibrating mount. The delay can be then scanned manually or electronically. The vibrating pot is controlled externally in amplitude and in speed with a frequency generator. The signal is measured via a photodetector sending the signal to an oscilloscope. Moreover, the autocorrelation can be use in intensity or interferometry configuration.

Concerning the other more standard equipment, it consists in a photodiode + oscilloscope $(500 \mathrm{MHz})$, a spectrometer and a power-meter (figure 3). The optics used for the beam transport, are specified to avoid any spectral phase distortions (silver mirrors typically). For group velocity dispersion a piece of SF18 glass and negative GVD mirrors from Layertec can be used.

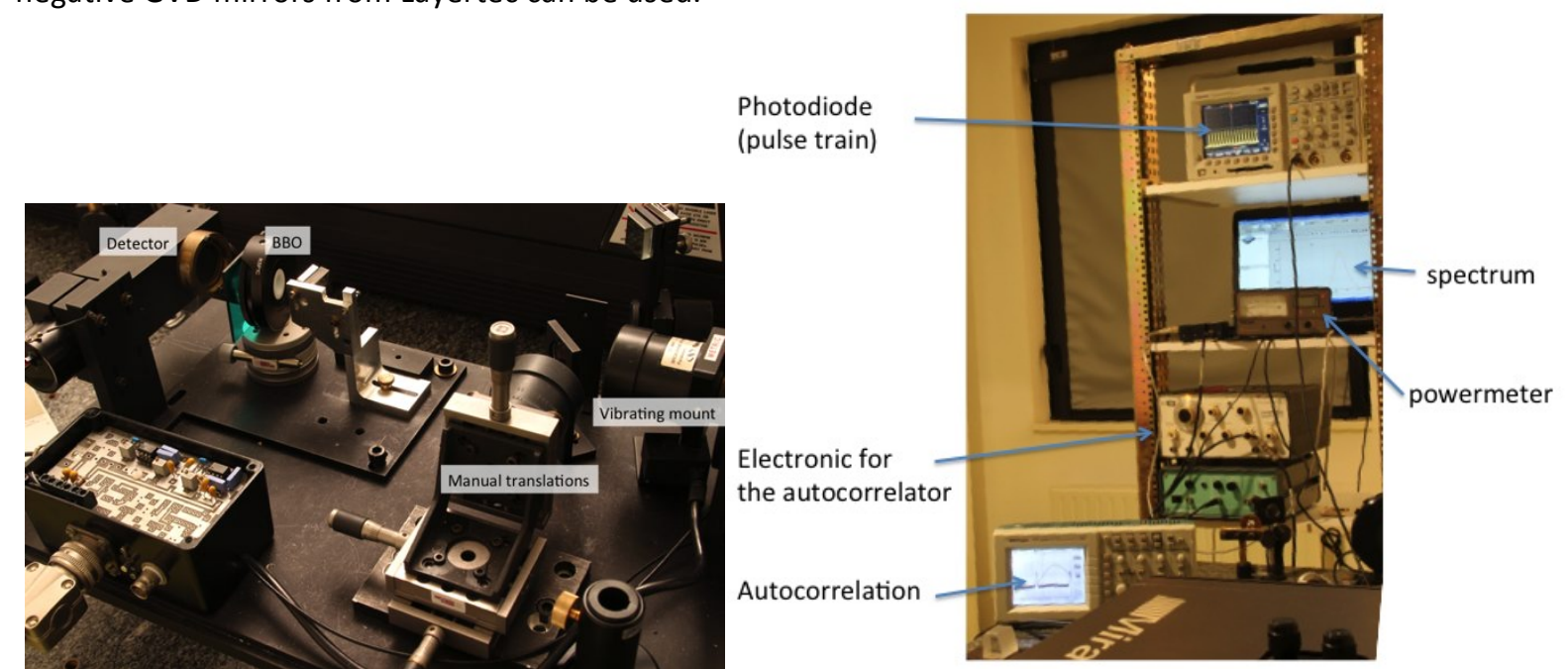

figure 3 : Diagnostics : (left) autocorrelator, (right) diagnostic screens

\section{b) On the Generation of the pulse train}

The first item discussed during the lab session is the demonstration of Kerr-Lens mode-locking (KLM). Through the manipulation, the student will understand the effect of the Kerr effect: the order of relatively small magnitude of this non-linear phase and the necessary exaltation of its impact being an important message to carry. This is the accurate handling by the student of a slit in the cavity. The locking of longitudinal modes in phase is also an essential concept. For this, a monitoring of the pulse train thanks to a fast photodiode, measuring the average power and spectrum display help to understand the different regimes in competition and discrimination. Indeed, different regimes from $\mathrm{cw}$ to stable mode-locking can be then observed and apprehended. Stability issues are also addressed modifying the wavelengths (and the associate laser gain) with an intracavity Lyot. 
The second part of the generation consists in understanding the solitonic effect. The soliton study is addressed by analyzing the interplay between self-phase modulation (SPM) and the intracavity group velocity dispersion (GVD). Measuring the pulse duration versus the intracavity GVD the theorem of the soliton area can also be apprehended figure 1 .

\section{c) On the measurement of the duration}

The lab work is developed in order to make students aware of basic measurement notions especially in ultrashort pulses field. Indeed, one must understand that the femtosecond-pulse characterization may not be managed with a fast photodiode whose bandwidth is typically in the $100 \mathrm{~s} \mathrm{MHz}$. The figure 4 brings a practical approach of a fast photodiode response than can be used to see stability issues. The photodiode is used as a tool for studying the stability of the mode-locked in different time scales.

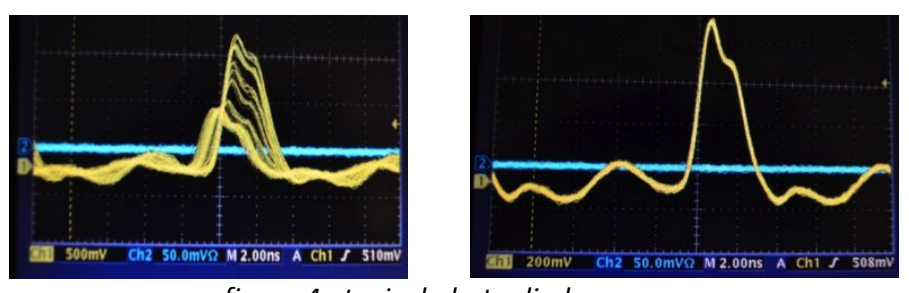

figure 4 : typical photo diode screens

Therefore, the measurement of the pulse width is achieved thanks to a fully accessible homemade autocorrelator. The displacement can be done manually and via a vibrating mount. The session text step-leads the student to find out the relation between the width of the autocorrelation and the width of the real pulse. The notion of deconvolution factor depending on the pulse shape (connected with the soliton study) is also approached

At least, the use of a spectrometer at the output of the laser permits approaching the time-bandwidth relation $\Delta t \Delta v \leq 1$. The broadening of the shape of the spectrum make the student think about the time-frequency relation.

\section{d) On the manipulation of the pulse}

At this point of the $4 \mathrm{~h} 30$ lab session, the students are able to generate and characterize ultra-short pulses. The aim of the next experiments concerns their manipulation. In order to introduce the notion of spectral phase, students send the fs pulses through a piece of $34 \mathrm{~mm}$ long SF18 glass (realized (Fig 2) at the Optics Laboratory). The measures spectra and autocorrelation are done with and without. The pulses are then stretched and can be recompressed through GTI mirrors (dispersion rebound -500 fs2). The goal is for the students to understand the interest of the control frequency chirp -which is used in amplified femtosecond systems (CPA) -. Moreover the dispersion coefficient $\left(\beta_{2}=154 \mathrm{fs}^{2} . \mathrm{mm}^{-1}\right.$ at $\left.800 \mathrm{~nm}\right)$ of the block introduced in the light pass may be retrieved.

\section{Conclusion}

This is the first time a femtosecond laser experiment is proposed to M2 students at the Institut d'Optique in France. It is axed on the three main issues which are generation, measure and control of ultrashort pulses. The strength of this labwork is its accessibility. Indeed, it has been designed such as the student truly handles the pulses from their generation to their control. Because this field is highly related to both courses and industrial issues, this lab work perfectly fits the training of future graduate students in Optics. 